\title{
A Two-Dimensional Weighting Function for a Driver Assistance System
}

\author{
Joost C. F. de Winter, Max Mulder, M. M. van Paassen, David A. Abbink, and Peter A. Wieringa, Member, IEEE
}

\begin{abstract}
Driver assistance systems that supply force feedback (FF) on the accelerator commonly use relative distance and velocity with respect to the closest lead vehicle in front of the own vehicle. This 1-D feedback might not accurately represent the situation and can cause unwanted step-shaped changes in the FFs during lateral maneuvers. To address these shortcomings, a 2-D system is proposed that calculates FF using a weighted average of the influences of lead vehicles. Offline simulations and an experiment in a driving simulator were performed to compare no feedback, 1-D systems, and the novel 2-D system during a car-following task with cut-in maneuvers. Results show that the 2-D feedback resulted in lower mean forces, lower response times to cut-in vehicles, and favorable subjective experiences as compared to the 1-D systems.
\end{abstract}

Index Terms-Accelerator, driver assistance, force feedback (FF), weighting function.

\section{INTRODUCTION}

C AR DRIVING is principally a visual task, with the view outside the car as the main source of information. When drivers allocate their attention elsewhere, e.g., inside the car to tune their radio, very little information remains about the driving situation, resulting in increased accident risk. Providing force feedback (FF) on the accelerator may help overcome this issue. Accelerator FF informs the driver of impending hazards, while preserving the driver's control over the accelerator. Previous studies have shown that - when using appropriate $\mathrm{FF}$ algorithms-car-following tasks can be performed with equal precision but with less accelerator movement [1], [2]. Moreover, through analyzing electromyographic activity of relevant muscles and modeling the human neuromusculoskeletal system, it was shown that part of the driver's control actions with the "haptic" gas pedal were performed by spinal

Manuscript received March 1, 2006; revised March 20, 2007. This paper was recommended by Associate Editor T. H. Lee.

J. C. F. de Winter is with the Department of Biomechanical Engineering, Faculty of Mechanical, Maritime and Materials Engineering, Delft University of Technology, 2628 CD Delft, The Netherlands (e-mail: J.C.F.deWinter@ tudelft.nl).

M. Mulder and M. M. van Paassen are with the Department of Control and Simulation, Faculty of Aerospace Engineering, Delft University of Technology, 2600 GB Delft, The Netherlands (e-mail: M.Mulder@tudelft.nl; M.M.vanPaassen@tudelft.nl).

D. A. Abbink is with the Haptics Laboratory, Department of Biomechanical Engineering, Faculty of Mechanical, Maritime, and Materials Engineering, Delft University of Technology, 2628 CD Delft, The Netherlands (e-mail: D.A.Abbink@tudelft.nl).

P. A. Wieringa is with the Faculty of Mechanical, Maritime, and Materials Engineering, Delft University of Technology, 2628 CD Delft, The Netherlands (e-mail: P.A.Wieringa@tudelft.nl).

Color versions of one or more of the figures in this paper are available online at http://ieeexplore.ieee.org.

Digital Object Identifier 10.1109/TSMCB.2007.908860 reflexes, resulting in faster responses and a reduction of visual workload [2].

The FF systems described in [1] and [2] operate in one dimension only. Feedback is based on the longitudinal distance and relative speed with respect to one-i.e., the closest-vehicle within range of the (virtual) sensor system. One-dimensional feedback can induce unwanted effects when more than one vehicle is ahead of the own vehicle. Step-shaped changes in the FFs have been found to occur during lateral maneuvers, such as cut-ins. Unwanted reflexes, excessive wear of hardware, and timing problems associated with the balance between false alarms and missed detections are likely disadvantages of feedback discontinuities [1], [3].

One problem of 1-D feedback can easily be remedied. By applying a filter, for example, limiting the rate of change of feedback, these feedback discontinuities cannot occur anymore. However, in doing so, feedback is still 1-D, which might not accurately represent the driver's perception of risk and could therefore lead to suboptimal driver comfort.

The goal of this paper is to develop and evaluate a solution for the limitations of 1-D feedback. A 2-D system is proposed that uses a weighted average of lead vehicles in calculating feedback. Indeed, the psychologically relevant aspects of car driving have often been represented in a 2-D fashion. Examples are the "field of safe travel" metaphor [4], a tube in which drivers operate [5], or a fan-shaped "committed zone" ahead of the own vehicle [6]. Two-dimensional potential-field methods have been proposed in the context of guiding vehicles [7]-[10]. We found these methods to be less suitable, however, because they either do not address the case of multiple vehicles or they superpose the influence of each vehicle, which leads to an overestimation of risk.

Our solution in calculating a weighted average can be interpreted as an intermediate solution between, on the one hand, the 1-D system that considers the lead vehicle having maximum importance, and on the other hand, existing 2-D systems that superpose the influences of all lead vehicles. In the following, our 2-D feedback system will be discussed and compared with some existing 1-D solutions.

\section{FF SYSTEMS}

Five FF solutions were evaluated: no feedback (NF), a 1-D system (1D), a rate-limited 1D system (1DR), a 2-D superposing system (2DS), and the weighted 2-D system (2DW). These five systems are described as follows.

NF A normal accelerator without FF is used. The driver can only rely on visual information. 
1D FF is applied according to (1), shown at the bottom of the next page. This equation is based on [11] and has been developed to correctly correspond with a driver's perception of risk. FF is a continuous function of the time headway (THW, in seconds), time-to-collision (TTC, in seconds), and throttle position ( $\alpha$ in percent). In this paper, THW is defined as the distance from the front bumper of the own vehicle to the rear bumper of the lead vehicle divided by the speed of the own vehicle. TTC is defined as the distance from the front bumper of the own vehicle to the rear bumper of the lead vehicle divided by the relative speed between the lead vehicle and the own vehicle (defined as positive if the gap closes). For 1D, THW and TTC are considered with respect to the closest lead vehicle in a 4-m-wide area extending to the front of the own vehicle.

1DR This is identical to $1 \mathrm{D}$ but, here, FF is rate-limited at $20 \mathrm{~N} / \mathrm{s}$. It can be considered a "quick-and-dirty" solution to prevent discontinuities in the FF. The limit of $20 \mathrm{~N} / \mathrm{s}$ was considered subjectively comfortable during exploratory runs with experienced drivers in a driving simulator.

2DS Here, all vehicles within the 4-m-wide area in front of the own car contribute to FF when being visible from the center of the front bumper of the own vehicle. FF is calculated for each lead vehicle and added together so that a resultant FF is obtained.

2DW A weighted average of THW and 1/TTC is calculated. Here, a weighted THW and weighted 1/TTC (rather than THW and 1/TTC with respect to only the nearest vehicle) are used to calculate FF, still using (1). The remainder of this section will be devoted in describing how vehicles are weighted.

The weight that is assigned to a particular vehicle depends on the position of its rear bumper in an egocentric weight field. The

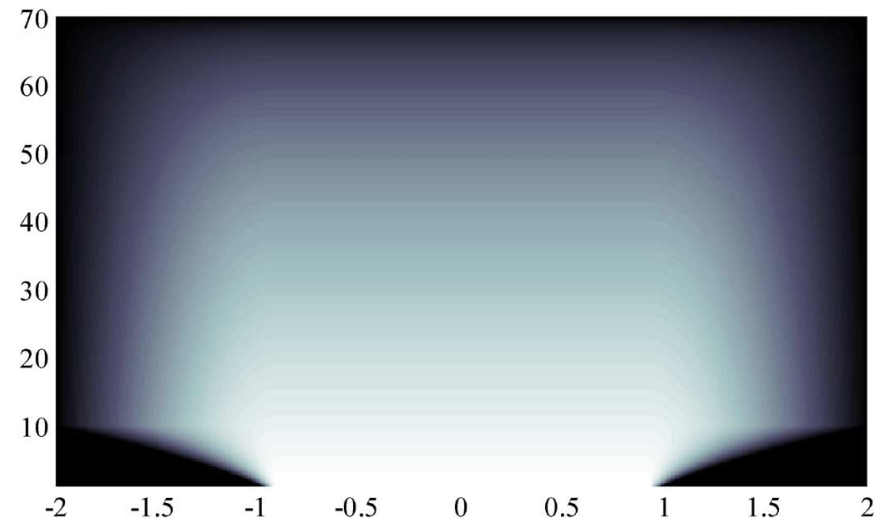

Fig. 1. Weight field for a speed of $100 \mathrm{~km} / \mathrm{h}$. The center of the own vehicle's front bumper is situated at $(0,0)$. A linear grayscale is used (black corresponds to zero weight, and white corresponds to maximum weight at $x=1 \mathrm{~m})$.

weight field is shown in Fig. 1 and is described mathematically in (2)-(5), shown at the bottom of this page. In (2)-(5), the following notations are used.

1) $x$ is the longitudinal road-fixed coordinate, in meters.

2) $y$ is the lateral road-fixed coordinate, in meters.

3) $W(x, y)$ is the weight for a particular coordinate.

4) $x_{b}$ is the longitudinal boundary of the weight field, in meters.

5) $y_{b}$ is the lateral boundary of the weight field, in meters.

6) $\theta$ is the angle of a coordinate with respect to the front bumper.

7) $r$ is half the own-vehicle width $(0.915 \mathrm{~m}$, in our case).

8) $p(=0.5)$ is a constant influencing the longitudinal weight distribution.

9) $V$ is the speed of the own vehicle, in meters per second.

10) $u$ is half the weight field width $(2.0 \mathrm{~m}$, in our case).

11) $s(=0.11 / \mathrm{s})$ and $t(=2.0 \mathrm{~m} / \mathrm{s})$ are constants that depend on the car dynamics and define the parabolic shape of the weight field.

$$
F F= \begin{cases}0, & \text { for }\left(\frac{1}{T H W}+\frac{8}{T T C}\right)<0.5 \\ 0, & \text { for } T T C \leq 0, T H W \leq 0 \\ 44.2, & \text { for }\left(\frac{1}{T H W}+\frac{8}{T T C}\right)>4.5 \\ (9.66+0.0771 \alpha) \cdot\left(\frac{1}{T H W}+\frac{8}{T T C}\right)^{0.898}, & \text { otherwise }\end{cases}
$$

$$
\begin{aligned}
W(x, y) & = \begin{cases}0, & \text { for }|y| \geq y_{b}, x \geq x_{b}, x \leq 0 \\
\left(x_{b}-x\right)^{p} \cdot \cos (\theta), & \text { for } r<|y|<y_{b} \\
\left(x_{b}-x\right)^{p}, & \text { for }|y| \leq r\end{cases} \\
\theta & =\frac{\pi}{2} \frac{\arctan ((|y|-r) / x)}{\arctan \left(\left(y_{b}-r\right) / x\right)} \\
x_{b} & =2.5 \cdot V \\
y_{b}(x) & = \begin{cases}r+(s / V) x^{2}+(t / V) x, & \text { for } r+(s / V) x^{2}+(t / V) x<u \\
u, & \text { for } r+(s / V) x^{2}+(t / V) x \geq u\end{cases}
\end{aligned}
$$




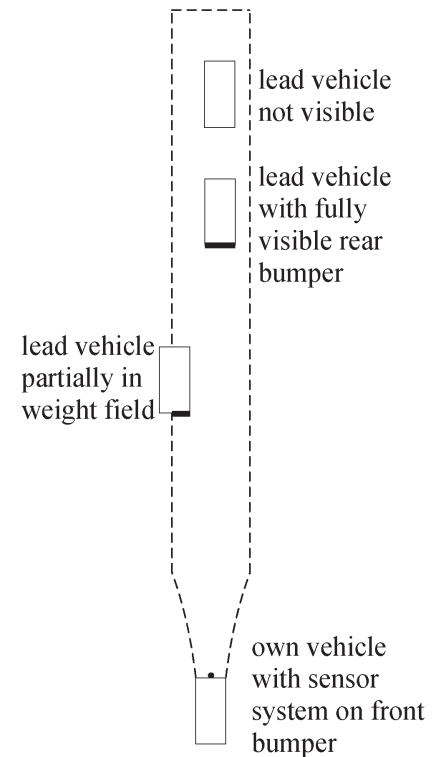

Fig. 2. Weighting of vehicles. Weight is assigned to (parts of) rear bumpers in the weight field that are in the line of sight of the center of the own vehicle's front bumper.

As shown in Fig. 2, parts of the rear bumpers of all vehicles in front, lying within the weight field and visible from the center of the own vehicle's front bumper, are included.

The weight field is continuous within its boundaries and is zero beyond. Hence, the weight assigned to vehicles moving within, into, or out of the weight field is always continuous, thereby avoiding discontinuous step-shaped changes in the FF during lateral maneuvers of the vehicles in front or of the own vehicle.

The broadening boundaries in front of the own vehicle (for longitudinal distances smaller than $10 \mathrm{~m}$ ) represent the contours of the reachable area. The width of the weight field was set at $4.0 \mathrm{~m}$. When assuming that lane keeping of both the own vehicle and lead vehicles is a normally distributed process with an average corresponding to the lane center and a standard deviation of 0.30 - and 3.6-m-wide lanes, this would result in a relatively low chance $(5.3 \%)$ that weight is assigned to a part of a vehicle passing on another lane. It can be seen in (4) that the weight field elongates for higher velocities. Thus, the weight distribution between two lead vehicles remains constant when following with constant THW at varying speeds.

\section{OfFline Simulations}

Offline simulations were performed to test the five alternatives for FF introduced above. A cut-in maneuver was simulated, as shown in Fig. 3. The own vehicle followed a lead vehicle at a THW of $1.25 \mathrm{~s}$, and a second lead vehicle cut-in from the left adjacent lane at a THW of $0.5 \mathrm{~s}$. Its lateral motion was sinusoidal, with a lane change time of $6 \mathrm{~s}$ [12] and a lane change distance of $3.6 \mathrm{~m}$. All lead vehicles had a length of $4.0 \mathrm{~m}$ and a width of $1.8 \mathrm{~m}$.

First, an open-loop simulation was performed which assumed that the driver keeps the accelerator at a constant position. The results (FF versus time) are shown in Fig. 4. The $\mathrm{FF}$ was equal for each condition during the beginning and at

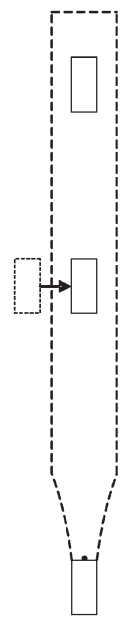

Fig. 3. Illustration of simulated cut-in maneuver.

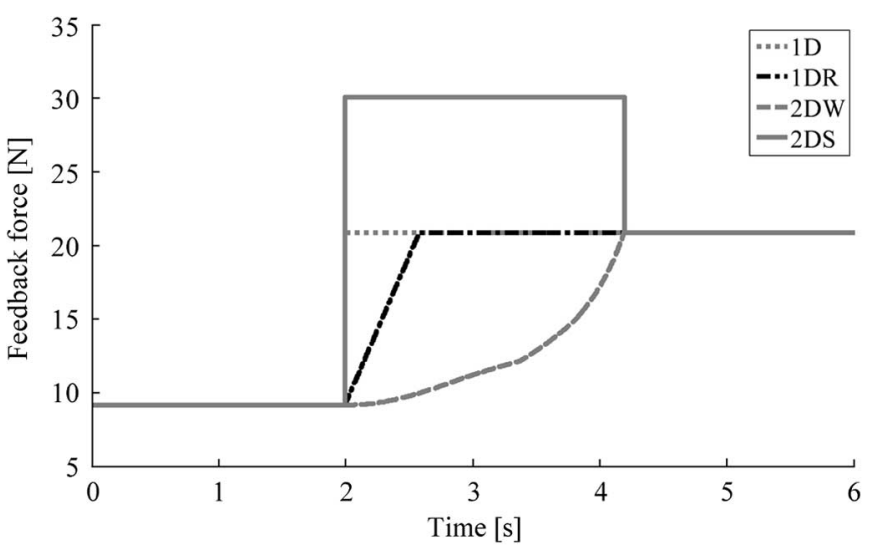

Fig. 4. FF versus time (open-loop simulation).

the end of the cut-in, because the systems function identically when only one vehicle is visible from the own vehicle's front bumper. For 1D, a sudden change in FF occurred at the moment the other lead vehicle became the closest vehicle within the 4-m-wide area. For 1DR, the change in feedback occurred at the same moment as with $1 \mathrm{D}$, but the rate of change is limited to $20 \mathrm{~N} / \mathrm{s}$. 2DS induced a high amount of feedback when both vehicles were visible, an undesirable effect as the forces on the pedal may become too large. 2DS has therefore been discarded in the remainder of this paper. As with $1 \mathrm{DR}$, the $2 \mathrm{DW}$ solution assured continuous feedback, i.e., no step-shaped changes were found. Feedback increased relatively late for $2 \mathrm{DW}$ as a result of the fact that it represents a weighted average of both lead vehicles. Mean FF was lowest for 2DW.

Second, a closed-loop simulation was performed using the same cut-in maneuver and feedback conditions as above (except for the 2DS). The driver was modeled as a linear controller with the goal to (visually) maintain a THW of $1.25 \mathrm{~s}$ and with a proportional control strategy (gain of 100, from weighted THW error in seconds to applied muscle force in newtons) and a time delay of $0.5 \mathrm{~s}$. FF was modeled to only passively influence the position of the driver's foot. The neuromuscular behavior of the foot was modeled as a mass (2.5 kg)-spring (200 Nm/rad)-damper $(5 \mathrm{Nm} \cdot \mathrm{s} / \mathrm{rad})$ system. The distance between the foot contact 


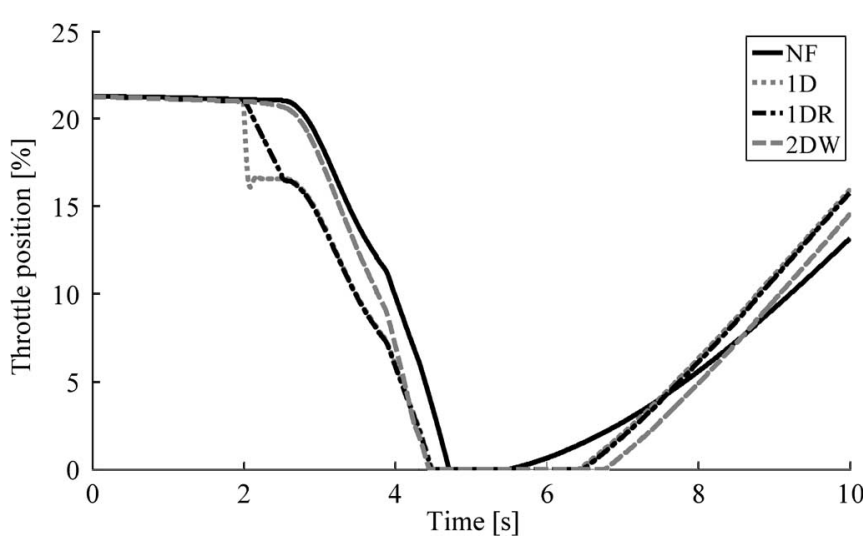

Fig. 5. Throttle position versus time (closed-loop simulation).

point at the accelerator and its rotation point was $0.20 \mathrm{~m}$. Throttle position, with a range of $20^{\circ}$, served as input to the longitudinal model of the own vehicle (mass of $1600 \mathrm{~kg}$, maximum engine power of $148 \mathrm{~kW}$ ). Initial speed was $100 \mathrm{~km} / \mathrm{h}$.

Results (throttle positions versus time since the start of the maneuver) are shown in Fig. 5. Around $2.0 \mathrm{~s}$, the cut-in vehicle entered the 4-m-wide area, resulting in an immediate response for 1D followed by a small oscillation attributable to the springlike properties of the foot, indicating that a stepshaped increase of FF indeed may cause an unwanted response. The initial responses for 2DW and NF were the slowest because FF lagged behind or was absent. At approximately $7 \mathrm{~s}$, the modeled driver depressed the accelerator to start following the cut-in vehicle.

\section{DRIVING-Simulator EXPERIMENT}

A driving-simulator experiment was performed to examine the actual human response to the feedback systems.

\section{A. Method}

Apparatus: The experiment was conducted in a fixed-base driving simulator, equipped with an actuated accelerator, a brake pedal, a steering wheel, and a digital dashboard display showing engine revolutions per minute and vehicle speed. The driving scene was projected on the laboratory wall, $2.9 \mathrm{~m}$ in front of the driver. The resolution of the projected image was $1024 \times 768$ pixels, and the size was $3.3 \mathrm{~m} \times 2.4 \mathrm{~m}$.

Subjects and Instructions: There were 14 subjects, 12 males and 2 females, aged 23 to 51, that participated. The mean duration of driver-license possession was 10.6 years, the mean selfestimated driving frequency per week was $4.4 \mathrm{~h}$, and the mean previous experience with FF in a driving simulator was $1.3 \mathrm{~h}$. Subjects were instructed through a written briefing, stating that they were about to test four accelerator systems, three of which featured FF. It was further stated that the three FF systems mainly differed during cut-ins. Subjects were instructed to remain on the right lane, to follow the preceding car at a THW of $1 \mathrm{~s}$, and to allow lead vehicles to cut-in from the left lane.

Independent Variables: The NF, 1D, 1DR, and 2DW FF systems introduced above were evaluated.
Experimental Design and Procedure: A within-subjects design was applied. Every subject drove four 12-min runs under each condition in randomized order. An 8-min training run was conducted before commencing the experiment.

Vehicle Model and Highway: An identical car model as in the offline simulations was used. A two-lane highway was simulated, with 3.60-m-wide lanes and with shoulders present on each side of the road. The road had left and right curves with an inner radii of $1000 \mathrm{~m}$.

Traffic: Other vehicles did not interact with the subject's vehicle. Mean speed of the other vehicles was $125 \mathrm{~km} / \mathrm{h}$ for the left lane and $100 \mathrm{~km} / \mathrm{h}$ for the right lane. For both lanes, longitudinal velocity (standard deviation (SD) of $8.3 \mathrm{~km} / \mathrm{h}$ ) was modeled based on available data from instrumented vehicles. The distance separation of the lead vehicles was based on a THW density distribution, with a mean THW of $1.15 \mathrm{~s}$, based on [13]. Lateral-positioning behavior (swerving) of the traffic was created according to a model based on available lanekeeping data (SD of $0.30 \mathrm{~m}$ ). In every run, at random, 21 of the lead vehicles passing on the left lane cut-in to the right lane. This cut-in vehicle then became the vehicle to be followed by the subject. Cut-ins took place along a trajectory in time defined by a Bezier spline. Three cut-in-maneuver types were used: "modal" (duration of $6 \mathrm{~s}$ ), "hazardous" (duration of $5 \mathrm{~s}$ ), and "very hazardous" (duration of $4 \mathrm{~s}$ ). Twelve of the 21 vehicles that cut-in to the right lane would cut-out to the left again; the other nine remained on the right lane.

Dependent Measures: A total of 44 performance measures were calculated for each run. Factor analysis was performed to cluster the measures into unique factors. This way, four useful factors were obtained. For each factor, two or three (intercorrelating) measures representing the factor were selected. Additionally, after each run, subjects were presented with questionnaires on a laptop computer. For each question, subjects could adjust a slider, which was divided into 20 equal intervals. The questionnaire included the NASA Task Load indeX (TLX) for assessing subjective workload [14] and four additional questions. All dependent measures are shown in Table I. Subjects had the opportunity to comment on the answers to the four additional questions in textboxes.

\section{B. Results}

One data file (subject ten, 1D condition) was corrupted and excluded from the analysis. Table II shows the results of the dependent measures for the four accelerator systems.

Braking and Steering Activity: Individual differences were relatively large with respect to braking and steering activity. However, no significant differences were observed between the four feedback conditions.

Following Distance and Precision: No significant differences were found between 2DW and the other conditions.

Throttle Activity: No significant differences were found for throttle activity. Throttle activity was slightly higher for NF as could be expected from earlier research [1].

Forces: The means and standard deviations of FF were significantly lower for 2DW as compared to $1 \mathrm{D}$ and $1 \mathrm{DR}$. 
TABLE I

DEPENDENT MEASURES

\begin{tabular}{|ll|}
\hline \multicolumn{2}{|l|}{ 1. Braking and Steering Activity } \\
\hline SD BrakeForce & \multicolumn{1}{|l|}{ SD of the brake pedal force (N) } \\
\hline SD Steer & SD of the steering wheel angle (deg) \\
\hline 2. Following Distance and Precision \\
\hline M THW & Mean THW to closest vehicle (m) \\
\hline SD THW & SD of THW to closest vehicle (m) \\
\hline 3. Throttle Activity & \\
\hline SD Throttle & SD of throttle position (\%) \\
\hline SD RPM & SD of engine speed (RPM) \\
\hline ThrottleReleased & Accelerator released ( $<$ 5\%) (\% of time) \\
\hline 4. Forces & \\
\hline M FF & Mean applied feedback force (N) \\
\hline SD FF & SD of applied feedback force (N) \\
\hline 5. Subjective Experience \\
\hline TLX & NASA Task Load Index score (5-100) \\
\hline Safety & (1-20) (very negative-very positive) \\
\hline Comfort & (1-20) (very negative-very positive) \\
\hline Pleasure & (1-20) (very negative-very positive) \\
\hline Correspond & $\begin{array}{l}\text { Correspondence between sensed forces and } \\
\text { visual perception (1-20) } \\
\text { (to a very low level-to a very high level) }\end{array}$ \\
\hline
\end{tabular}

TABLE II

RESUlts FOR THE FOUR FEEDBACK CONDITIONS

\begin{tabular}{|l|c|c|c|c|l|}
\hline & NF & 1D & 1DR & 2DW & Significance \\
\hline Braking and Steering Activity (mean of subjects) \\
\hline SD BrakeForce (N) & 35.5 & 31.6 & 35.6 & 32.7 & \\
\hline SD Steer (deg) & 1.05 & 1.04 & 1.05 & 1.04 & \\
\hline Following Distance and Precision (mean of subjects) \\
\hline M THW (m) & 1.37 & 1.53 & 1.49 & 1.48 & \\
\hline SD THW (m) & 0.48 & 0.55 & 0.52 & 0.49 & \\
\hline Throttle Activity (mean of subjects) \\
\hline SD Throttle (\%) & 25.6 & 24.5 & 24.5 & 24.5 & \\
\hline SD RPM (RPM) & 495 & 453 & 460 & 456 & \\
\hline ThrottleReleased (\%) & 25.7 & 24.8 & 23.8 & 24.1 & \\
\hline Forces (mean of subjects) & $\mathrm{X}$ & 11.9 & 11.7 & 10.3 & $*(1 \mathrm{D}, 1 \mathrm{DR})$ \\
\hline M FF & $\mathrm{X}$ & 6.7 & 5.4 & 4.3 & $* * *(1 \mathrm{D}, 1 \mathrm{DR})$ \\
\hline SD FF & 36 & 50 & 47 & 42 & $*(1 \mathrm{D})$ \\
\hline Subjective Experience (median & of subjects) \\
\hline TLX (5-100) & 9 & 8.5 & 10 & 12.5 & $*$ (NF,1D) \\
\hline Safety (1-20) & 9.5 & 7 & 6.5 & 13.0 & $*(1 \mathrm{D}, 1 \mathrm{DR})$ \\
\hline Comfort (1-20) & 11 & 7.5 & 8 & 11 & \\
\hline Pleasure (1-20) & 1 & 7.5 & 11 & 15 & $* * *(\mathrm{NF})$ \\
\hline Correspond (1-20) & 1 & \\
\hline
\end{tabular}

Means of subjects were evaluated by a repeated measures analysis of variance (ANOVA). When equal variances could not be assumed, medians were evaluated by a Friedman test. ${ }^{* * *} p<0.001, * 0.01 \leq p<0.05$. A TukeyKramer multiple comparison was performed to evaluate whether 2DW differed from other conditions at a $95 \%$ confidence level (shown between parentheses).

Subjective Experience: Subjective workload (TLX), safety, and comfort were more favorable for $2 \mathrm{DW}$ as compared to 1D, a significant effect. The correspondence between FF and visual information was rated highest for $2 \mathrm{DW}$. Analysis of the written comments revealed that five of 14 subjects had reported that 2DW felt comfortable as compared to two subjects who reported this characteristic for the other conditions.

There are 11 subjects who drove with $1 \mathrm{D}$ that reported that they had received feedback that they could not explain or feedback from the wrong car, particularly from overtaking vehicles driving on the other lane. Three subjects reported the 1D feedback to be "aggressive" or "discontinuous." NF yielded relatively low scores on the TLX and good scores on

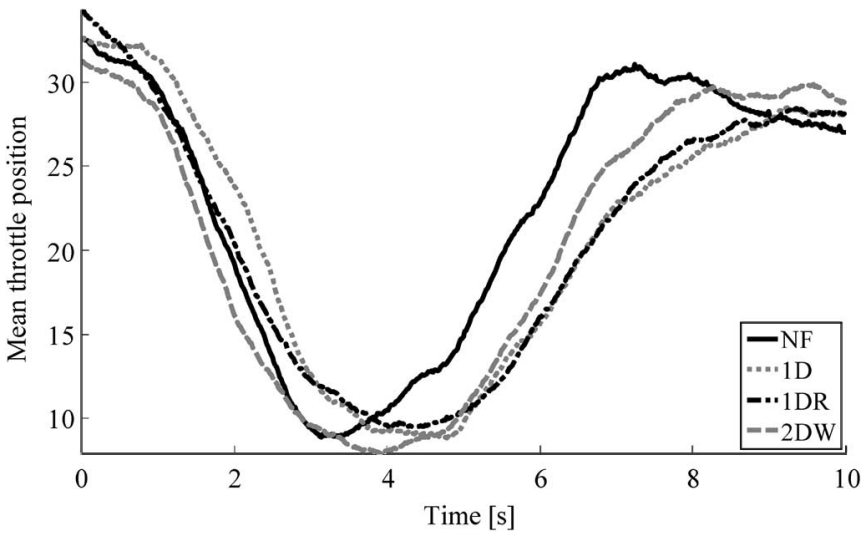

Fig. 6. Averaged throttle position versus time (driving-simulator experiment).

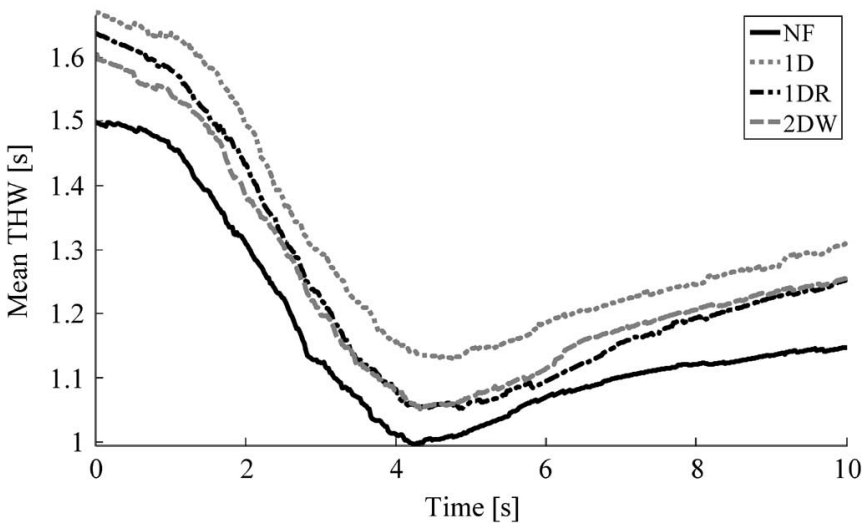

Fig. 7. Averaged THW versus time (driving-simulator experiment).

the subjective pleasure question. Four subjects reported to be satisfied at being in full control in the absence of any feedback. Five subjects reported to be annoyed at not being in full control when driving with FF.

The responses to cut-ins were analyzed in further detail because the conditions only differ from each other when multiple vehicles are considered. Fig. 6 shows the averaged throttle position of all cut-ins versus time since the start of the cut-in. Subjects released the accelerator soonest for 2DW. A repeatedmeasures analysis of variance (ANOVA) with Tukey-Kramer multiple comparison indicated that the mean throttle position at $2 \mathrm{~s}$ after the start of the cut-in was significantly lower for 2DW as compared to 1D $(p=0.01)$. Fig. 7 shows the averaged THW of all cut-ins. It can be seen that THW is lowest for NF and highest for 1D. The mean THW of 1DR and 2DW closely resemble. Fig. 8 shows the averaged FF. As could be expected from the simulations, FFs were lower and increased later for 2-DW as compared to 1D and 1DR.

\section{CONCLUSION}

In this paper, a 2-D FF system was proposed that calculates feedback using a weighted average of vehicles in front of the own vehicle. It provides a solution to what are considered the two main problems with conventional 1-D approaches. First, it eliminates the occurrence of discontinuous accelerator 


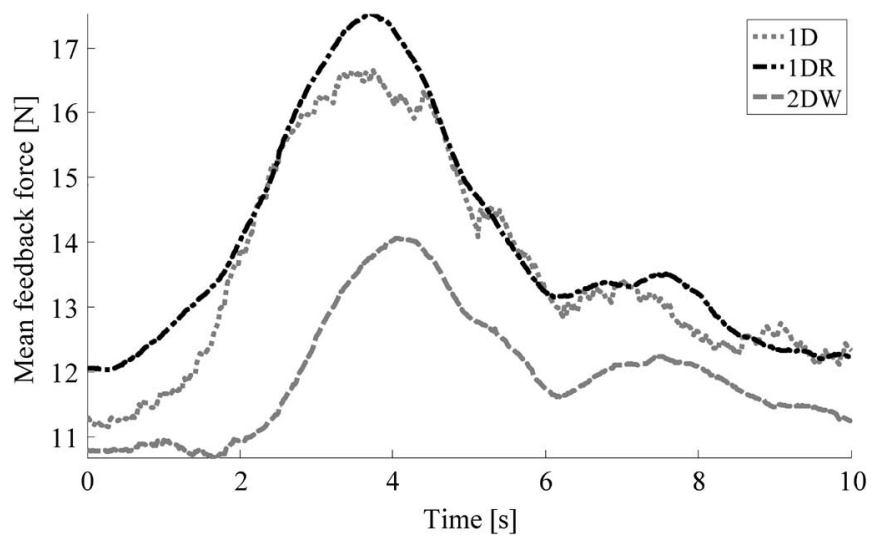

Fig. 8. Averaged FF versus time (driving-simulator experiment).

movements that are caused by sudden changes of what the system considers to be the lead vehicle. Second, as it is based on a weighted contribution of all lead vehicles, depending on their relative risk, its behavior better matches the driver's expectations. This research can be applied to also improve other 1-D systems, such as adaptive cruise control. It is recommended to investigate whether the concept of "risk weighting" in providing FF can be generalized to other applications, like teleoperation.

\section{REFERENCES}

[1] M. Mulder, "Haptic gas pedal feedback for active car-following support," Ph.D. dissertation, Dept. Aerosp. Eng., Technische Univ. Delft, Delft, The Netherlands, 2007.

[2] D. A. Abbink, "Neuromuscular analysis of haptic gas pedal feedback during car following," Ph.D. dissertation, Dept. Mech., Maritime and Mater. Eng., Technische Univ. Delft, Delft, The Netherlands, 2006.

[3] J. C. F. de Winter, M. Mulder, M. M. van Paassen, and T. Yamamura, "Weighted two-dimensional longitudinal impedance for driving support system," in Proc. IEEE Int. Conf. Syst., Man, and Cybern., The Hague, The Netherlands, pp. 256-260.

[4] J. J. Gibson and L. E. Crooks, "A theoretical field-analysis of automobiledriving," Amer. J. Psychol., vol. 51, no. 3, pp. 453-471, Jul. 1938.

[5] H. Summala, "Hierarchical model of behavioural adaptation and traffic accidents," in Traffic and Transport Psychology, T. Rothengatter and E. Carbonell Vaya, Eds. Amsterdam, The Netherlands: Pergamon, 1997, pp. 41-52.

[6] S. F. Hulbert and A. Burg, "Human factors in transportation systems," in Systems Psychology, K. B. De Greene, Ed. New York: McGraw-Hill, 1970, pp. 471-509.

[7] D. Reichardt and J. Schick, "Collision avoidance in dynamic environments applied to autonomous vehicle guidance on the motorway," in Proc. IEEE Intell. Veh. Symp., Paris, France, 1994, pp. 74-78.

[8] H. W. Boschloo, T. M. Lam, M. Mulder, and M. M. van Paassen, "Collision avoidance for a remotely-operated helicopter using haptic feedback," in Proc. IEEE Int. Conf. Syst., Man, and Cybern., The Hague, The Netherlands, 2004, pp. 229-235.

[9] M. P. Hennessey, C. Shankwitz, and M. Donath, "Sensor based "virtual bumpers" for collision avoidance: Configuration issues," in Proc. SPIE, Dec. 1995, vol. 2592, pp. 48-59.

[10] J. C. Gerdes, E. J. Rossetter, and U. Saur, "Combining lanekeeping and vehicle following with hazard maps," Veh. Syst. Dyn., vol. 36, no. 4/5, pp. 391-411, Nov. 2001

[11] T. Kondoh, T. Yamamura, S. Kitazaki, N. Kuge, and E. R. Boer, "Identification of visual cues and quantification of drivers' perception of proximity risk to the lead vehicle in car-following situations," in Proc. Driving Simul. Conf. Asia/Pac., Tsukuba, Japan, 2006.

[12] E. C. B. Olsen, S. E. Lee, W. W. Wierwille, and M. J. Goodman, "Analysis of distribution, frequency, and duration of naturalistic lane changes," in Proc. Hum. Factors and Ergon. Soc. 46th Annu. Meeting, Baltimore, MD, 2002, pp. 1789-1793.
[13] L. Neubert, L. Santen, A. Schadschneider, and M. Schreckenberg, "Single-vehicle data of highway traffic: A statistical analysis," Phys. Rev. E, Stat. Phys. Plasmas Fluids Relat. Interdiscip. Top., vol. 60, no. 6, pp. 6480-6490, Dec. 1999.

[14] S. G. Hart and L. E. Staveland, "Development of NASA-TLX (task load index): Results of empirical and theoretical research," in Human Mental Workload, P. A. Hancock and N. Meshkati, Eds. Amsterdam, The Netherlands: North Holland, 1988, pp. 139-183.

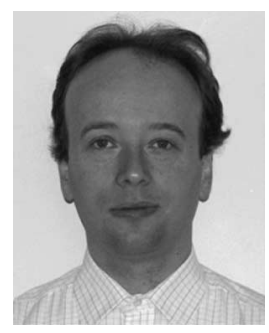

Joost C. F. de Winter was born in Utrecht, The Netherlands, on March 7, 1979. He received the M.Sc. degree in aerospace engineering, specializing in control and simulation, from the Delft University of Technology, Delft, The Netherlands, in 2004. He is currently working toward the Ph.D. degree in the Department of Biomechanical Engineering, Faculty of Mechanical, Maritime and Materials Engineering, Delft University of Technology.

His research interests include simulation-based driver training, driver assessment, and driver assistance systems.

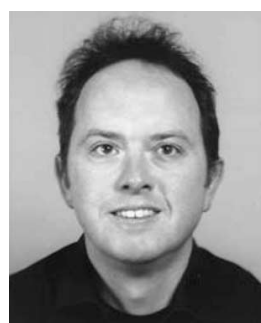

Max Mulder received the M.Sc. and Ph.D. (cum laude) degrees in aerospace engineering from the Delft University of Technology, Delft, The Netherlands in 1992 and 1999, respectively. His Ph.D. dissertation focused on the cybernetics of tunnel-in-the-sky displays.

$\mathrm{He}$ is currently an Associate Professor with the Department of Control and Simulation, Faculty of Aerospace Engineering, Delft University of Technology. His research interests include cybernetics and its use in modeling human perception and performance, and cognitive systems engineering and its application in the design of ecological human-machine interfaces.

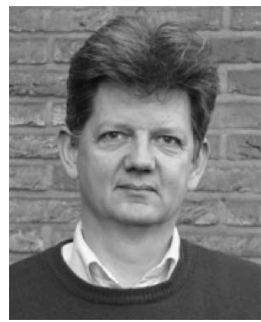

M. M. van Passen received the M.Sc. and Ph.D. degrees from the Delft University of Technology, Delft, The Netherlands, in 1988 and 1994 respectively. His Ph.D. research was the study of the role of the neuromuscular system of the pilot's arm in manual control.

For two years, he was a Brite/EuRam Research Fellow with the University of Kassel, Kassel, Germany, with Prof. Johannsen, and a half year at the Technical University of Denmark, Copenhagen, Denmark, where he worked on advanced interfaces for process control. He is currently an Associate Professor with the Department of Control and Simulation, Faculty of Aerospace Engineering, Delft University of Technology, working on aircraft simulation and human-machine interaction. His work on human-machine interaction ranges from studies of perceptual processes and human manual control to complex cognitive systems. In the latter field, he applies cognitive systems engineering analyses (abstraction hierarchy, multilevel flow modeling) and ecological interface designs to vehicle control. 


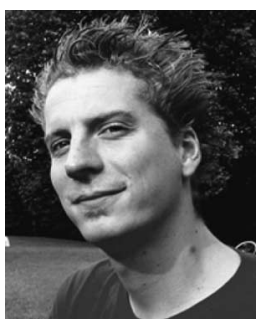

David A. Abbink was born in Purmerend, The Netherlands, on September 19, 1977. He received the M.Sc. degree in mechanical engineering and the Ph.D. degree from the Delft University of Technology, Delft, The Netherlands, in 2002 and 2006, respectively. His Ph.D. dissertation was "Neuromuscular Analysis of Haptic Feedback during Car Following," for which he received the 2006 Best Dutch Thesis in Movement Sciences.

For three years, he worked on a research project funded by Nissan, which aimed at developing a haptic driver support system. He currently holds a postdoctoral position with the Haptics Laboratory, Department of Biomechanical Engineering, Faculty of Mechanical, Maritime and Materials Engineering, Delft University of Technology, focusing his research on neuromuscular analysis and the human-centered design of haptic guidance for various applications.

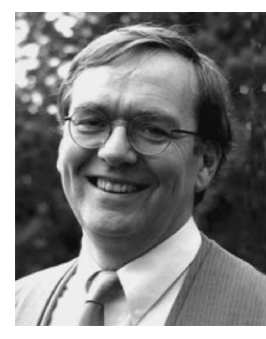

Peter A. Wieringa (M'90) received the M.Sc. and $\mathrm{Ph} . \mathrm{D}$. degrees (cum laude) from the Delft University of Technology (DUT), Delft, The Netherlands, in 1980 and 1985, respectively. His Ph.D. dissertation was the investigation of blood and oxygen distribution in a capillary network model of the heart muscle.

From 1987 to 1991 he was a Fellow of the Royal Dutch Academy of Sciences. From 1988 to 1990, he was trained in microvascular research with the University of Virginia, Charlottesville. He continued this research with DUT and the University of Amsterdam, Amsterdam, The Netherlands. In 1991, he became an Associate Professor of man-machine systems and studied human supervisory behavior and the reliability of complex systems, including medical systems, with DUT. Since 2000, he has been with the Faculty of Mechanical, Maritime and Materials Engineering (formerly the Faculty of Design and Engineering), where he was a Full Professor and Head of the Department of Medical Technology and Mechanics from December 2000 to 2006 and is currently the Director of Studies and Vice Dean.

Dr. Wieringa received an International Fogarty Fellowship from the U.S National Institutes of Health in 1988. 